\title{
Fractional Fourier approximations for potential gravity waves on deep water
}

\author{
V. P. Lukomsky and I. S. Gandzha \\ Department of Theoretical Physics, Institute of Physics, Prospect Nauky 46, Kyiv 03028, Ukraine
}

Received: 6 May 2003 - Revised: 15 August 2003 - Accepted: 2 September 2003

\begin{abstract}
In the framework of the canonical model of hydrodynamics, where fluid is assumed to be ideal and incompressible, waves are potential, two-dimensional, and symmetric, the authors have recently reported the existence of a new type of gravity waves on deep water besides well studied Stokes waves (Lukomsky et al., 2002b). The distinctive feature of these waves is that horizontal water velocities in the wave crests exceed the speed of the crests themselves. Such waves were found to describe irregular flows with stagnation point inside the flow domain and discontinuous streamlines near the wave crests.

In the present work, a new highly efficient method for computing steady potential gravity waves on deep water is proposed to examine the character of singularity of irregular flows in more detail. The method is based on the truncated fractional approximations for the velocity potential in terms of the basis functions $1 /\left(1-\exp \left(y_{0}-y-i x\right)\right)^{n}, y_{0}$ being a free parameter. The non-linear transformation of the horizontal scale $x=\chi-\gamma \sin \chi, 0<\gamma<1$, is additionally applied to concentrate a numerical emphasis on the crest region of a wave for accelerating the convergence of the series. For lesser computational time, the advantage in accuracy over ordinary Fourier expansions in terms of the basis functions $\exp (n(y+i x))$ was found to be from one to ten decimal orders for steep Stokes waves and up to one decimal digit for irregular flows. The data obtained supports the following conjecture: irregular waves to all appearance represent a family of sharp-crested waves like the limiting Stokes wave but of lesser amplitude.
\end{abstract}

\section{Introduction}

From old times the wave motion of the ocean bewitched and extremely attracted the attention of mankind. Up to now the problem of understanding specific features of water waves and their modelling represent a real challenge both from scientific and engineering points of view. Occurrence of ex-

Correspondence to: V. P. Lukomsky (lukom@iop.kiev.ua) tremely large and steep ocean breaking waves imposes a hazard to fishing boats, ships, and off-shore oil facilities. To understand physical mechanisms that give rise to extreme breaking waves and to model them correctly it is necessary to gain detailed knowledge of the form and dynamics of steep water waves.

The canonical problem about the propagation of surface waves on deep water (see Sect. 2) was the first essentially non-linear problem in hydrodynamics. Its analysis during almost two hundred years gave the origin to many fields of non-linear dynamics such as solitary waves, modulation instabilities, strange attractors, etc. Stokes (1847) was the first who considered surface waves of finite amplitude (Stokes waves). Small amplitude waves are sinusoidal. As the wave amplitude grows, the crests become steeper and sharper whilst the troughs flatten. Stokes (1880) conjectured that such waves must have a maximal amplitude (the limiting wave) and showed the flow in this wave to be singular at the crest forming a $120^{\circ}$ corner (the Stokes corner flow). Much later, Grant (1973) suggested that this singularity, for a wave that has not attained the limiting form, is located above the wave crest and forms a stagnation point with streamlines meeting at right angles. Longuet-Higgins and Fox (1978) proved this numerically after extending Stokes flows analytically outside the domain filled by fluid. The following question resulted: why the flow in the limiting Stokes wave has the $120^{\circ}$ singularity instead of the $90^{\circ}$ one, as in any wave with lesser amplitude? Because of this Grant (1973) conjectured that a continuous approach to the limiting amplitude is possible only if the Stokes corner flow has several coalescing singularities. However, it has not yet clear where these multiple singularities arise from.

A new era in developing the theory of steep gravity waves started from the work of Longuet-Higgins (1975), where he found that many characteristics of gravity waves, such as speed, energy, and momentum, are not monotonic functions of the wave amplitude, as was assumed from Stokes, but attain total maxima and then drop before the limiting wave is reached. Longuet-Higgins and Fox (1977) constructed 
asymptotic expansions for waves close to the $120^{\circ}$-cusped wave (almost highest waves) and showed that these dependences oscillate infinitely as the limiting wave is approached. Nevertheless, strict numerical verification of such oscillations seems to be a real challenge up to the present time, with only the first relative maximum and minimum having been thoroughly investigated (see, e.g. Longuet-Higgins and Tanaka, 1997).

Tanaka (1983) showed that gravity waves steeper than the wave with maximal total energy become unstable with respect to two-dimensional disturbances having the same period as an undisturbed wave (superharmonic instability). Jillians (1989) investigated the form of such instabilities and showed that they lead to wave overturning and breaking. The conjecture was made that wave breaking is a purely local phenomenon around the wave crest which, in the case of spilling breakers and more gently plunging breakers, occurs independently of the flow in the rest of a wave. Proceeding with this idea Longuet-Higgins and Cleaver (1994) and Longuet-Higgins et al. (1994) suggested that superharmonic instability results in the crests of almost highest Stokes waves to be unstable (crest instability). Longuet-Higgins and Tanaka (1997) strongly supported the conclusion that superharmonic instabilities of Stokes waves are indeed crest instabilities. Finally, Longuet-Higgins and Dommermuth (1997) showed that crest instabilities lead (i) to wave overturning and breaking or (ii) to a smooth transition of a wave to a lower progressive wave having nearly the same total energy, followed by a return to a wave of almost the initial wave height. The latter fact generated a new question: what is the nature of such a transient phenomenon? A possible explanation would be found if superharmonic instability resulted in a bifurcation to a new solution, as usually takes place in nonlinear dynamics. However, up to this time it was assumed that the Stokes solution is unique and free of bifurcations in keeping with the uniqueness argument of Garabedian (1965). The only bifurcation known to occur is the trivial one of a pure phase shift at the point of energy maximum (Tanaka, 1985).

The above results are all related to Stokes waves, for which the speed of fluid particles at the wave crests is smaller than the wave phase speed, equality being achieved for the limiting wave only. Thus, the traditional criterion for wave breaking is that horizontal water velocities in the crest must exceed the speed of the crest (Banner and Peregrine, 1993). Lukomsky et al. (2002a,b) have recently provided evidence (although numerical and not completely rigorous) for the existence of a new family of two-dimensional irrotational symmetric periodic gravity waves that satisfy the criterion of breaking. A stagnation point in the flow field of these waves is inside the flow domain, in contrast to the Stokes waves of the same wavelength. This makes streamlines exhibit discontinuity in the vicinity of the wave crests, with near-surface particles being jetted out from the flow. Because of this such waves and flows were called irregular (in contrast to regular Stokes flows).

To calculate irregular flows Lukomsky et al. (2002a,b) used truncated Fourier expansions for the velocity potential and the elevation of a free surface in the plane of spatial variables (a physical plane). Debiane and Kharif (see Gandzha et al., 2002) confirmed the existence of irregular waves using inverse plane Longuet-Higgins method (Longuet-Higgins, 1986), where the spatial coordinates are represented as Fourier series in velocity potential and stream function, the corresponding coefficients being evaluated by solving quadratic relations between them. Finally, Clamond (2003) also obtained irregular flows by applying his new renormalized cnoidal wave (RCW) approximation. In spite of this progress, irregular waves at present are only approximate and not enough accurate numerical solutions. The following question has to be answered then: what are the form and properties of irregular wave when its numerical error vanishes, that is, what real physical solutions do irregular waves approximate?

Ordinary Fourier expansions used by Lukomsky et al. (2002a) become not efficient enough for approximating irregular waves and even Stokes waves close to the limiting one due to slow descending of the Fourier coefficients. Thus, a more efficient method is necessary for these tasks. Up to now the most precise and efficient way for calculating the properties of two-dimensional surface waves is Tanaka's method of the inverse plane (see Tanaka, 1983, 1986). The key idea of his method is to map the inverse plane into a unit circle by means of the Nekrasov transformation. Then boundary conditions are transformed to an integral equation, which is solved iteratively. The accuracy of obtained solutions is drastically improved by concentrating a numerical emphasis on the crest region using further transformation of variables. As a result, Tanaka's method is the only one being capable of evaluating the second maximum of the phase speed and even further higher order extremums. In spite of all the advantages of Tanaka's method and his program, where it is implemented, we are interested in improving the methods of the physical plane since they can be applied for calculating 3-D waves as well and can be generalized to the case of non-ideal and compressible fluid, in contrast to all the inverse plane methods.

Thus, the purpose of this paper is to present a new method in the physical plane for calculating two-dimensional potential steady progressive surface waves on the fluid of infinite depth (see Sect. 3). The method is based on the fractional Fourier approximation for the velocity potential recently introduced by the authors (Gandzha et al., 2002; Lukomsky et al., 2002c) and the non-linear transformation of the horizontal scale for concentrating a numerical emphasis near the wave crest. The first term of such a fractional Fourier approximation was independently derived by Clamond (2003) and was called a renormalized cnoidal wave approximation.

In Sect. 4, fractional Fourier approximations are applied for calculating regular and irregular flows. In Sect. 4.1, great numerical advantage of fractional approximations over ordinary Fourier approximations is demonstrated when calculating almost highest Stokes waves. Although the accuracy of the results is still less than the ones obtained us- 
ing Tanaka's method, proposed fractional Fourier approximations have a potential to become almost as effective as the method of Tanaka. An additional set of stagnation points is found to exist above the crest area of Stokes waves supporting the conjecture of Grant (1973) that the $120^{\circ}$ singularity of the limiting wave is formed of several coalescing $90^{\circ}$ singularities. In Sect. 4.2, the profiles of irregular waves are demonstrated to reveal the Gibbs phenomenon usually taking place when a discontinuous function or a continuous function with discontinuous derivatives are approximated by continuous truncated Fourier series. Moreover, even regular Stokes waves very close to the Stokes corner flow are also demonstrated to exhibit the similar Gibbs phenomenon in accordance with the observation of Chandler and Graham (1993). The data presented resulted in the following assumption: irregular waves are very likely to approximate a family of sharp-crested waves like the limiting Stokes wave but of lesser amplitude. Concluding remarks are given in Sect. 5.

\section{The canonical model}

Consider the dynamics of steady potential two-dimensional periodic waves on the irrotational, inviscid, incompressible fluid with unknown free surface under the influence of gravity. Waves are assumed to propagate without changing their form from left to right along the $x$-axis with constant speed $c$ relative to the motionless fluid at infinite depth (see Fig. 1). Gravity waves and related fluid flows are governed by the following set of equations

$$
\begin{aligned}
\Phi_{\theta \theta}+\Phi_{y y}=0, & -\infty<y<\eta(\theta) ; \\
\left(c-\Phi_{\theta}\right)^{2}+\Phi_{y}^{2}+2 \eta=c^{2}, & y=\eta(\theta) \\
\left(c-\Phi_{\theta}\right) \eta_{\theta}+\Phi_{y}=0, & y=\eta(\theta) \\
\Phi_{\theta}=0, & \Phi_{y}=0, \quad y=-\infty
\end{aligned}
$$

where $\theta=x-c t$ is the wave phase, $\Phi(\theta, y)$ is the velocity potential (the velocity is equal to $\vec{\nabla} \Phi), \eta(\theta)$ is the elevation of the unknown free surface, and $y$ is the upward vertical axis such that $y=0$ is the still water level. Herein Eq. (1) is the Laplace equation in the flow domain, Eq. (2) is the dynamical boundary condition (the Bernoulli equation, $c^{2}$ is the Bernoulli constant), Eq. (3) is the kinematic boundary condition (no fluid crosses the surface), Eq. (4) is the condition that fluid is motionless at infinite depth. The dimensionless variables are chosen such that length and time are normalized by the wavenumber $k$ and the frequency $\sqrt{g k}$ of a linear wave, respectively, $g$ being the acceleration due to gravity. In this case, the dimensionless wavelength $\lambda=2 \pi$.

When the total mass of the fluid is assumed to remain unchanged the wave mean level coincides with the still water level, that is, $\bar{\eta}=0$, the overdash designating averaging over the wave period. The Bernoulli equation then results in the Levi-Civita relation $\overline{q^{2}}=0$, where $q^{2}$ is the squared velocity at the free surface in the frame of reference moving together with the wave (the wave related frame of reference).

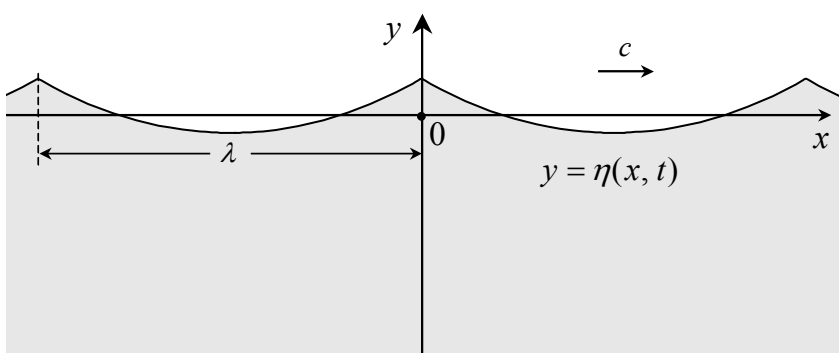

Fig. 1. The laboratory frame of reference.

Once the velocity potential and the wave phase speed are known, particle trajectories in the wave related frame of reference (streamlines) are found from the following differential equations:

$\frac{\mathrm{d} \theta}{\mathrm{d} t}=\Phi_{\theta}(\theta, y)-c, \quad \frac{\mathrm{d} y}{\mathrm{~d} t}=\Phi_{y}(\theta, y) ;$

Each streamline is characterized by a constant value of a stream function $\psi(\theta, y)$ in the wave related frame of reference. The velocity potential and the stream function $\Psi(\theta, y)=\psi(\theta, y)+c y$ in the laboratory frame of reference are connected by means of the Cauchy-Riemann conditions:

$\Phi_{\theta}=\Psi_{y} ; \Phi_{y}=-\Psi_{\theta}$.

This makes possible introducing the complex potential $W=$ $\Phi+i \Psi$ so that

$\Phi=-i c\left(R-R^{*}\right), \quad \Psi=c\left(R+R^{*}\right), \quad R_{\theta}=i R_{y} ;$

where $R=i W^{*} / 2 c,{ }^{*}$ is the complex conjugate. In terms of the complex function $R(\theta, y)$, the dynamical and kinematic boundary conditions (2), (3) are as follows:

$$
\begin{gathered}
i c^{2}\left(R_{\theta}-R_{\theta}^{*}\right)+2 c^{2} R_{\theta} R_{\theta}^{*}+\eta=0, \quad y=\eta(\theta) ; \\
R(\theta, \eta)+R^{*}(\theta, \eta)-\eta=0 .
\end{gathered}
$$

Since the velocity potential and the stream function are defined to within an arbitrary constant the integration constant in Eq. (9) is included into the stream function to make $\psi=0$ at the free surface. Then $\left.\bar{\Psi}\right|_{y=\eta(\theta)}=c \bar{\eta}=0$ and the stream function at infinite depth $\left.\Psi\right|_{y=-\infty}=c \bar{\eta}-I$, where

$$
I=\frac{1}{2 \pi} \int_{0}^{2 \pi} \mathrm{d} \theta \int_{-\infty}^{\eta(\theta)} \Phi_{\theta}(\theta, y) \mathrm{d} y=\left.\bar{\Psi}\right|_{y=\eta(\theta)}-\left.\Psi\right|_{y=-\infty} .
$$

is the wave impulse averaged over the period. The quantity $K=I / c$ is the mass flux transferred by a wave over the period and is called the Stokes flow.

In addition to the Laplace equation and the boundary conditions, an initial condition should be assigned. Since the canonical model is energy conservative, the wave total energy can be used instead to characterise wave properties. For this purpose, however, the crest-to-trough height $H$ or the wave steepness $A=H / \lambda$ are the more convenient parameters since they monotonously increase starting from linear waves up to the limiting configuration. Thus, using the 
Eqs. (1), (8), (9), (4) of the canonical model the following quantities are to be found as the functions of the wave steepness $A$ : the complex function $R(\theta, y)$, the elevation $\eta(\theta)$ of the free surface, and the wave phase speed $c$.

\section{The method for obtaining solutions}

\subsection{Fractional Fourier approximations}

When working in the plane of spatial variables the solutions to the Laplace equation (1) in the flow domain are usually looked for as the following truncated Fourier series

$R(\theta, y)=\sum_{n=0}^{N} \xi_{n} \exp (n(y+i \theta))$.

This approach was applied by the authors (Lukomsky et al., 2002a,b) for calculating steep gravity waves. Fourier expansions (10), however, become ineffective for steep waves with sharpening crests close to the limiting wave due to slow descending of the Fourier coefficients. Because of this we proposed (Gandzha et al., 2002; Lukomsky et al., 2002c) a more effective set of functions to expand the velocity potential on the basis of the following Euler formula (see Hamming, 1962)

$$
\begin{aligned}
& \sum_{n=1}^{\infty} \sigma_{n} z^{n}= \sum_{n=1}^{\infty} \frac{\zeta_{n}}{\left(1-z^{-1}\right)^{n}}, \\
& \zeta_{n}=\sum_{n_{1}=1}^{n}(-1)^{n_{1}} C_{n-1}^{n_{1}-1} \sigma_{n_{1}},
\end{aligned}
$$

$C_{n}^{n_{1}}$ being the binomial coefficients. By choosing $z(\theta, y)=$ $\exp \left(y-y_{0}+i \theta\right) ; \sigma_{n}=\xi_{n} \exp \left(n y_{0}\right)$ the following oneparametric expansion for the velocity potential is obtained after truncating the series:

$$
\begin{aligned}
R\left(\theta, y ; \quad y_{0}\right) & =\sum_{n=0}^{N} \frac{\zeta_{n}}{\left(1-\exp \left(y_{0}-y-i \theta\right)\right)^{n}} \\
& =\sum_{n=0}^{N} \frac{\alpha_{n}}{\left(\exp \left(-y_{0}\right)-\exp (-y-i \theta)\right)^{n}} \\
& \equiv \sum_{n=0}^{N} \alpha_{n} T^{n}\left(\theta, y ; y_{0}\right), \\
T\left(\theta, y ; y_{0}\right) & =\left(\exp \left(-y_{0}\right)-\exp (-y-i \theta)\right)^{-1},
\end{aligned}
$$

where the normalized coefficients $\alpha_{n}=\zeta_{n} \exp \left(-n y_{0}\right)$ were introduced to overcome infinite exponents at $y_{0} \rightarrow \infty$; $\alpha_{0} \equiv \xi_{0}$. Approximation (12) shows a formal correspondence with Padé-type fractional approximates. Because of this we called expansion (12) a "fractional Fourier expansion". It is singular in a countable number of isolated points $y=y_{0}, \theta=2 \pi k, k \in \mathbb{Z}$, their location being determined by a free parameter $y_{0}$. Singular points are to be located outside the flow domain for calculating potential waves. At $y_{0}=\infty$, fractional Fourier expansion (12) reduces to ordinary Fourier expansion (10) with $\xi_{n}=(-1)^{n} \alpha_{n}$. Due to
Eq. (11), expansions (10) and (12) are equivalent at $N=\infty$ and the convergence of (12) follows from the convergence of Eq. (10). For finite $N$ and $y_{0} \sim 1$, however, a fractional Fourier expansion converges much more rapidly than an ordinary Fourier expansion. The reason is that a finite number of terms in Eq. (12) always corresponds to an infinite number of terms in Eq. (10) that is especially important for waves with sharpening crests.

The zero constant term in expansions (12) and (10) is defined by the value of the stream function at infinite depth:

$\alpha_{0} \equiv \xi_{0}=\left.\frac{1}{2 c} \Psi\right|_{y=-\infty}=\frac{1}{2}(\bar{\eta}-K)$.

Expansions (12), (10), and, in general, any function $R(\theta, y)=R(y+i \theta)$ all satisfy the Laplace equation (1) exactly. The latter fact was also used by Clamond (1999, 2003) (for finite and infinite depth, respectively) to introduce a renormalization principle that allows reconstructing the velocity potential in the whole domain once the velocity potential at the bottom (or any other level) is known. By applying such renormalization to the first-order periodic solution of KdV equation Clamond (2003) obtained the velocity potential being exactly the same to the first term $(N=1)$ of expansion (12), which he called a renormalized cnoidal wave (RCW) approximation. There may be other possibilities to improve ordinary Fourier expansion (10) besides the proposed fractional expansion (12). However, one should additionally assure the convergence of series that makes constructing such generalized expansions much more difficult.

One can see from the expansion of derivatives

$$
\begin{aligned}
R_{y}\left(\theta, y ; y_{0}\right) & =-i R_{\theta}=\sum_{n=1}^{N+1} \beta_{n} T^{n}\left(\theta, y ; y_{0}\right), \\
\beta_{n} & =n \alpha_{n}-(n-1) \alpha_{n-1} \exp \left(-y_{0}\right),
\end{aligned}
$$

which follows directly from Eq. (12), that the boundary condition at infinite depth (Eq. 4) is also satisfied exactly.

Hereafter, only the symmetric waves are considered. In this case, the coefficients $\alpha_{n}$ and $\xi_{n}$ are real (in general, they are complex numbers for nonsymmetric waves). After taking into account expansions (12) and (15) the boundary conditions (8), (9) at the free surface attain the following form:

$$
\begin{aligned}
& 2 c^{2}\left(\sum_{n_{1}=1}^{N+1} \beta_{n_{1}} \operatorname{Re}\left(T^{n_{1}}\right)-\right. \\
& \left.-\sum_{n_{1}=1}^{N+1} \sum_{n_{2}=1}^{N+1} \beta_{n_{1}} \beta_{n_{2}} \operatorname{Re}\left(T^{n_{1}} T^{* n_{2}}\right)\right)=\eta, \quad y=\eta(\theta) ; \\
& 2 \sum_{n_{1}=0}^{N} \alpha_{n_{1}} \operatorname{Re}\left(T^{n_{1}}\right)-\eta=0, \quad y=\eta(\theta) .
\end{aligned}
$$

Note that Eqs. (16) and (17) at $N \rightarrow \infty$ are equivalent to boundary conditions (8) and (9), in the class of $2 \pi$-periodic functions (subharmonic waves with multiple periods are not taken into account in expansions 12). 


\subsection{Nonlinear transformation of the horizontal scale}

To solve boundary conditions (16) and (17) one should assign an appropriate approximation to the unknown elevation $y=$ $\eta(\theta)$ of the free surface. In the plain of spatial variables, the Fourier series

$\eta(\theta)=\sum_{n=-\infty}^{\infty} \eta_{n} \exp (\operatorname{in} \theta), \quad \eta_{-n}=\eta_{n}$

are often used. Note that the collocation method can be used instead but it is less efficient than expansion (18) (see Lukomsky et al., 2002b). The mean level $\bar{\eta}=\eta_{0}$ should be zero for exact solutions. For approximate solutions (when the series are truncated), $\eta_{0}$ becomes nonzero due to Levi-Civita relation not being held exactly and can be used to estimate the precision of approximate results.

Adequate description of sharpening profiles close to the limiting one requires taking into account excessively large number of modes due to extremely slow descending of the Fourier coefficients. This highly restricts practical application of Eq. (18). The following non-linear transformation of the horizontal scale originally suggested by Chen and Saffman (1980)

$\theta(\chi ; \gamma)=\chi-\gamma \sin \chi, \quad 0<\gamma<1$,

allows overcoming this difficulty by stretching wave crests to a more rounded configuration. As a result, the Fourier series

$\eta(\chi ; \gamma)=\sum_{n=-M}^{M} \eta_{n}^{(\gamma)} \exp (i n \chi), \quad \eta_{-n}^{(\gamma)}=\eta_{n}^{(\gamma)}$

in the $\chi$-space with stretched crests are much more efficient (Lukomsky et al., 2002c). Due to nonlinear transformation (Eq. 19) any finite number $M$ of the coefficients $\eta_{n}^{(\gamma)}$ at $\gamma \neq 0$ corresponds to infinite number of the coefficients $\eta_{n} \equiv \eta_{n}^{(0)}$ in ordinary Fourier series $(\gamma=0)$, the associated relations being presented in Appendix A. Thus, the role of the parameter $\gamma$ for the series (Eq. 18) in horizontal coordinate $\theta$ is the same to the role of the parameter $y_{0}$ for the series (Eq. 10) in vertical coordinate $y$.

\subsection{Numerical procedure}

By means of Eq. (20) the boundary conditions (16), (17) at the free surface are reduced to the following system of nonlinear algebraic equations

$$
\begin{array}{r}
\mathcal{D}_{n}=c^{2} d_{n}-\eta_{n}^{(\gamma)}=0, \quad n=\overline{0, M} ; \\
\mathcal{K}_{n}=2 \sum_{n_{1}=1}^{N} \alpha_{n_{1}} t_{n}^{\left(n_{1}\right)}-\eta_{n}^{(\gamma)}=0, \quad n=\overline{1, N} ;
\end{array}
$$

where

$$
d_{n}=2 \sum_{n_{1}=1}^{N+1} \beta_{n_{1}}\left(t_{n}^{\left(n_{1}\right)}-\sum_{n_{2}=n_{1}}^{N+1} \beta_{n_{2}}\left(2-\delta_{n_{1}, n_{2}}\right) t_{n}^{\left(n_{1}, n_{2}\right)}\right) \text {, }
$$

$\delta_{n_{1}, n_{2}}$ is the Kronecker delta. The coefficients $t_{n}^{\left(n_{1}\right)}$ and $t_{n}^{\left(n_{1}, n_{2}\right)}$ are the Fourier harmonics of the functions $\operatorname{Re}\left(T^{n_{1}}\right)$ and $\operatorname{Re}\left(T^{n_{1}} T^{* n_{2}}\right)$, respectively:

$$
\begin{aligned}
t_{n}^{\left(n_{1}\right)} & =\frac{1}{2 \pi} \int_{0}^{2 \pi} \operatorname{Re}\left(T^{n_{1}}(\theta(\chi), \eta(\chi))\right) \exp (-i n \chi) \mathrm{d} \chi, \\
t_{n}^{\left(n_{1}, n_{2}\right)} & =\frac{1}{2 \pi} \int_{0}^{2 \pi} \operatorname{Re}\left(T^{n_{1}} T^{* n_{2}}\right) \exp (-i n \chi) \mathrm{d} \chi .
\end{aligned}
$$

They were calculated using the fast Fourier transform (FFT). The zero term $\alpha_{0}$ and, therefore, the Stokes flow $K$ are found from the kinematic equations (22) at $n=0$ :

$\alpha_{0}=\frac{1}{2} \eta_{0}^{(\gamma)}-\sum_{n_{1}=1}^{N} \alpha_{n_{1}} t_{0}^{\left(n_{1}\right)}, \quad K=\bar{\eta}-2 \alpha_{0}$.

The truncation of Eqs. (21), (22) was chosen for the following reasons. Since the set of kinematic equations (22) is linear over the coefficients $\alpha_{n}(n=\overline{1, N})$, they can be found in terms of the harmonics $\eta_{n}^{(\gamma)}$ without using dynamical equations (21). To proceed in such a way, it is sufficient to take into account only the first $N$ kinematic equations. Then the rest $M+1$ variables $c, \eta_{0}^{(\gamma)}, \eta_{n}^{(\gamma)}(n=\overline{2, M})$ are found from dynamical equations (21). The last unknown parameter $\eta_{1}^{(\gamma)}$ is determined by the wave steepness $A=(\eta(0)-\eta(\pi)) / 2 \pi$ as follows

$\eta_{1}^{(\gamma)}=\frac{\pi}{2} A-\sum_{n=1}^{[(M-1) / 2]} \eta_{2 n+1}^{(\gamma)}$

the square brackets designating the integer part. Since the wave steepness $A$ is an integral characteristic, some wave properties may be missed when using it as a governing parameter. Thus, we additionally use the first harmonic $\eta_{1}$ of the elevation in the $\theta$-space (a spectral characteristic) as an independent variable instead of the wave steepness. In this case, the first harmonic $\eta_{1}^{(\gamma)}$ in the $\chi$-space is expressed in terms of $\eta_{1}$ and the rest of the harmonics $\eta_{n}^{(\gamma)}(n=\overline{2, M})$ by means of the expression (A1) at $n=1$ instead of Eq. (25).

The set of Eqs. (21), (22) was solved by Newton's method, the Jacoby matrix being given in Appendix B. Starting values for new calculations were taken from previous runs. For large enough $N$ and $M$, the Jacoby matrix was found to become badly conditioned. Because of this the program realization was implemented in arbitrary precision computer arithmetic. For instance, computations at $N=150, M=2.5 N$ demand 160-digit arithmetic that is ten times more accurate than the machine one. Note that such a run is equivalent in computer time to a run with $N=250, M=4 N$ using ordinary Fourier approximations and because of this takes approximately 4 times lesser computer memory.

The truncation numbers $N$ and $M$ are chosen for the following reasons. By fixing the number $N$ in expansion (12) an approximate configuration of the velocity potential is assigned. To find out a proper truncation of the series (Eq. 20) 
for the elevation associated with this configuration, the number $M$ should be increased until the revision of solutions for greater $M$ becomes less than chosen accuracy. Then the precision to which boundary conditions (9) and (8) are satisfied defines the absolute errors connected with a truncation of the potential and elevation, respectively. Absolute errors of the dynamical and kinematic conditions divided by the constant terms contained in these equations, that is, the Bernoulli constant $c^{2}$ and the Stokes flow $K$, respectively, produce the corresponding relative errors. The overall relative error $E r_{\max }$ of an approximate solution is agreed to be the maximal relative error in boundary conditions (8) and (9) all over the wave period. To obtain a solution close to the exact one, one should gradually increase the number $N$, choosing every time a proper value of $M$, until overall desired precision is achieved. In a majority of calculations, it was sufficient to use the approximation $M=2.5 \mathrm{~N}$ or lesser ones.

The numerical scheme proposed operates with two parameters $y_{0}$ and $\gamma$. Decreasing $y_{0}$ from $y_{0}=\infty$ to $y_{0} \sim 1$ accelerates the convergence of the fractional Fourier expansion (12) for the velocity potential, lesser $N$ being necessary to retain the same accuracy. Increasing $\gamma$ from $\gamma=0$ to $\gamma=1-\varepsilon, \varepsilon \rightarrow 0$ accelerates the convergence of the expansion (20) for the elevation, lesser $M$ being necessary to retain the same accuracy. These two processes, however, should be carried out simultaneously. Using the fractional Fourier expansion (12) without the transformation of the horizontal scale (19) was found to deteriorate the convergence of series (18) and, vice versa, using the transformation of the horizontal scale without the fractional Fourier expansion was found to deteriorate the convergence of expansion (10), with only slight overall benefit having been achieved. On the contrary, using the fractional Fourier expansion in combination with the nonlinear transformation of the horizontal scale proved to be highly efficient (see Sect. 4).

\subsection{Physical quantities}

Once the coefficients $\alpha_{n}, \eta_{n}^{(\gamma)}$ and the wave phase speed $c$ are found, a variety of wave characteristics can be calculated. The velocity potential, stream function, and the horizontal and vertical velocities of fluid particles are as follows:

$$
\begin{aligned}
\Phi(\theta, y) & =2 c \sum_{n=0}^{N} \alpha_{n} \operatorname{Im}\left(T^{n}(\theta, y)\right) ; \\
\Psi(\theta, y) & =2 c \sum_{n=0}^{N} \alpha_{n} \operatorname{Re}\left(T^{n}(\theta, y)\right) ; \\
\Phi_{\theta}(\theta, y) & =2 c \sum_{n=1}^{N+1} \beta_{n} \operatorname{Re}\left(T^{n}(\theta, y)\right) ; \\
\Phi_{y}(\theta, y) & =2 c \sum_{n=1}^{N+1} \beta_{n} \operatorname{Im}\left(T^{n}(\theta, y)\right) ; \\
\beta_{n} & =n \alpha_{n}-(n-1) \alpha_{n-1} \exp \left(-y_{0}\right) .
\end{aligned}
$$

The horizontal and vertical accelerations of fluid particles are as follows:

$$
\begin{aligned}
& \frac{\mathrm{d}^{2} \theta}{\mathrm{d} t^{2}}=\Phi_{\theta \theta}\left(\Phi_{\theta}-c\right)+\Phi_{y \theta} \Phi_{y}, \\
& \frac{\mathrm{d}^{2} y}{\mathrm{~d} t^{2}}=\Phi_{y \theta}\left(\Phi_{\theta}-c\right)-\Phi_{\theta \theta} \Phi_{y} ;
\end{aligned}
$$

where

$$
\begin{aligned}
\Phi_{\theta \theta}(\theta, y) & =-2 c \sum_{n=1}^{N+2} \mu_{n} \operatorname{Im}\left(T^{n}(\theta, y)\right) ; \\
\Phi_{y \theta}(\theta, y) & =2 c \sum_{n=1}^{N+2} \mu_{n} \operatorname{Re}\left(T^{n}(\theta, y)\right) ; \\
\mu_{n} & =n \beta_{n}-(n-1) \beta_{n-1} \exp \left(-y_{0}\right) .
\end{aligned}
$$

The Stokes flow $K$, the wave impulse $I$, and the wave kinetic energy $E_{\mathrm{Kin}}$ are as follows (see Cokelet, 1977, for kinetic energy):

$K=\eta_{0}-2 \alpha_{0}, \quad I=K c, \quad E_{\mathrm{Kin}}=c I / 2$

$\alpha_{0}$ and $\eta_{0}$ being determined from relations (14) and (A1), respectively.

The wave potential energy $U$ is calculated as follows:

$$
\begin{aligned}
U= & \frac{1}{2 \pi} \int_{0}^{2 \pi} \frac{1}{2} \eta^{2}(\chi) \mathrm{d} \theta=\frac{1}{2}\left(\eta_{0}^{(\gamma)}\right)^{2}+\sum_{n_{1}=1}^{M}\left(\eta_{n_{1}}^{(\gamma)}\right)^{2}- \\
& \frac{\gamma}{2}\left(\eta_{0}^{(\gamma)} \eta_{1}^{(\gamma)}+\sum_{n_{1}=1}^{M} \eta_{n_{1}}^{(\gamma)}\left(\eta_{n_{1}-1}^{(\gamma)}+\eta_{n_{1}+1}^{(\gamma)}\right)\right) .
\end{aligned}
$$

\section{Regular and irregular flows}

\subsection{Stokes flows}

The dependence $c(A)$ of the phase speed of almost highest Stokes waves on their steepness calculated using fractional Fourier approximations is shown in Fig. 2 by the branch 1-23-6. And the corresponding dependence $c\left(\eta_{1}\right)$ of the phase speed on the first harmonic of the elevation is presented in Fig. 3 by the branch 1-2-3-4-6. The corresponding points of extremums in phase speed $c$, the first harmonic $\eta_{1}$, and steepness $A$ are presented in Table 1 .

The advantage of fractional Fourier approximations over ordinary Fourier approximations is well seen from Table 2. There, the values of the wave phase speed $c$ and the mean water level $\eta_{0}$ calculated using these two approaches are presented at different values of the wave steepness $A$ up to the limiting value. The deviation of $\eta_{0}$ from zero provides an estimation of the precision of approximate results. The maximal relative errors $E r_{\text {max }}$ of corresponding approximate solutions are also presented for analysis. One can see from the relative errors that the benefit from using fractional Fourier approximations with parameters chosen varies from 
ten decimal orders for $A=0.14(\approx 99.25 \%$ of the limiting steepness) to one decimal order for $A=0.141064$ (almost the limiting steepness). After taking into account that a run using fractional Fourier approximations with $N=120$, $M=2.5 N$ needs approximately 2.5 times lesser computer time and approximately 10 times lesser computer memory than a run using ordinary Fourier approximations with $N=$ 250, $M=4 N$, the advantage of fractional approximations becomes doubtless.

Nevertheless, the results presented for the values of steepness beyond the first minimum of $c(A=0.14092)$ are still less accurate than the ones obtained from Tanaka's program, which are also included into Table 2 for comparison. This is also well seen from Fig. 2, where the second maximum of $c(A=0.141056$, the point 5$)$ was obtained only using Tanaka's program. The fractional Fourier approximation at $y_{0}=0.9$ is sufficient to obtain the second maximum of $\eta_{1}$ (the point 4 in Fig. 3), but not sufficient to trace the second maximum of $c$. The reason is that the value $y_{0}=0.9$ used is optimal for the steepness corresponding to the first minimum of $c$, yet lesser $y_{0}$ being necessary for greater $A$ to improve the precision of fractional Fourier approximations. However, using the present computer realization of the method the authors failed to accomplish this task due to unsatisfactory convergence of their numerical algorithm for $y_{0}<0.9$. If this problem could be resolved fractional Fourier approximations in the physical plane would have a potential to become almost as effective as Tanaka's method in the inverse plane.

The flow field in Stokes waves is regular, that is, fluid particles move slower than the wave itself all over the flow domain. In the Stokes corner flow only, the fluid particle at the wave crest moves with velocity equal to the wave phase speed and, therefore, is motionless with respect to the wave. Because of this such points in the flow field are called the stagnation points. For all the Stokes waves other than the limiting one, a stagnation point is located outside the flow domain, as was at first shown by Grant (1973). The examples of such regular flows mapped outside the domain filled by fluid are presented in Fig. 4 for $A=0.14092$ and $A=0.14103$. The streamlines coming to/from the stagnation point $O$ (the separatrices) meet at right angles in accordance with the results of Grant (1973) and Longuet-Higgins and Fox (1978). This is the general rule provided that the stagnation point and the wave crest do not merge (see Appendix $\mathrm{C}$ for details). One can see from Fig. 4 that as the wave steepness is increased from $A=0.14092$ to $A=0.14103$ the wave crest becomes sharper, the stagnation point $O$ and the crest approaching each other. The downward and upward horn-like separatrices incoming to and outcoming from the stagnation point $O$, respectively, become steeper and attain the vertical tangent closer to the vertical axis. In the limiting case, when the stagnation point and the wave crest completely merge, these two separatrices should come together and form a vertical line, where the upward and downward streamlines coincide. This provides a simple illustration how a vertical cut of the complex plane in the Stokes corner flow shown in Fig. D1 (see Appendix D) is formed.
It is not clear, however, how a $120^{\circ}$ corner at the crest of the limiting Stokes wave is continuously formed from a $90^{\circ}$ singularity that is inherent any flow at lesser amplitude. In view of this, Grant (1973) suggested that a $120^{\circ}$ singularity should be formed from several coalescing singularities. The flow field shown in Fig. 4 at $A=0.14103$ provides an insight where these multiple singularities arise from. One can see that two additional symmetric $90^{\circ}$ stagnation points $O_{r}$ and $O_{l}$ exist above the wave crest at some distance from the vertical axis. These lateral stagnation points also exist at $A=0.14092$ (and apparently at any lesser steepness) but are located outside the plot region in Fig. 4. Moreover, the points $O_{r}$ and $O_{l}$ are only the first ones in a whole set of similar stagnation points located almost equidistantly in horizontal coordinate and having almost the same vertical position at fixed $A$. As the wave steepness is increased, the points $O_{r}$ and $O_{l}$ move towards the central stagnation point $O$, the distance between all the stagnation points decreasing. Note that although the flow field in the domain filled by fluid and the position of the stagnation point $O$ in Fig. 4 are accurate enough, numerical accuracy sharply drops in the region, where the stagnation points $O_{r}$ and $O_{l}$ are located. The flow field in this area has not been stabilized yet with respect to improving accuracy. As numerical accuracy is increased at fixed $A$, the lateral stagnation points all move towards the vertical axis, their vertical position remaining almost unchanged. Therefore, they may finally settle down at the vertical axis above the stagnation point $O$. Further investigation is necessary to verify this assumption. Nevertheless, the existence of a set of additional stagnation points, which approach the central stagnation point $O$ as the steepness is increased, makes us expect that a $120^{\circ}$ singularity in the Stokes corner flow is indeed formed from several (probably an infinite number of) coalescing $90^{\circ}$ singularities supporting the conjecture of Grant (1973).

\subsection{Irregular flows}

Lukomsky et al. (2002b) have numerically revealed a new type of flows, where fluid particles move faster than the wave itself in the vicinity of the wave crest due to the stagnation point located inside the flow domain. Because of this such flows and waves were called irregular.

Irregular waves can be traced continuously from regular Stokes waves in the following way. It is more natural to suggest that the point of the maximal (limiting) steepness $A_{\max }$, where the Stokes branch breaks (the point 6 in Fig. 2), is a turning point of the dependence $c(A)$ rather than a breaking point as was assumed before. To proceed to a new branch (which we called irregular) emanating from the point $A_{\max }$ one should simply use another governing parameter that does not have an extremum at the turning point, e.g. the first harmonic $\eta_{1}$ of the wave profile (the wave speed $c$ and many other parameters can be used as well). By tuning this parameter continuously starting from almost limiting Stokes waves one automatically proceeds to the irregular branch via the limiting point 6 as is seen from the dependence $c\left(\eta_{1}\right)$ in 

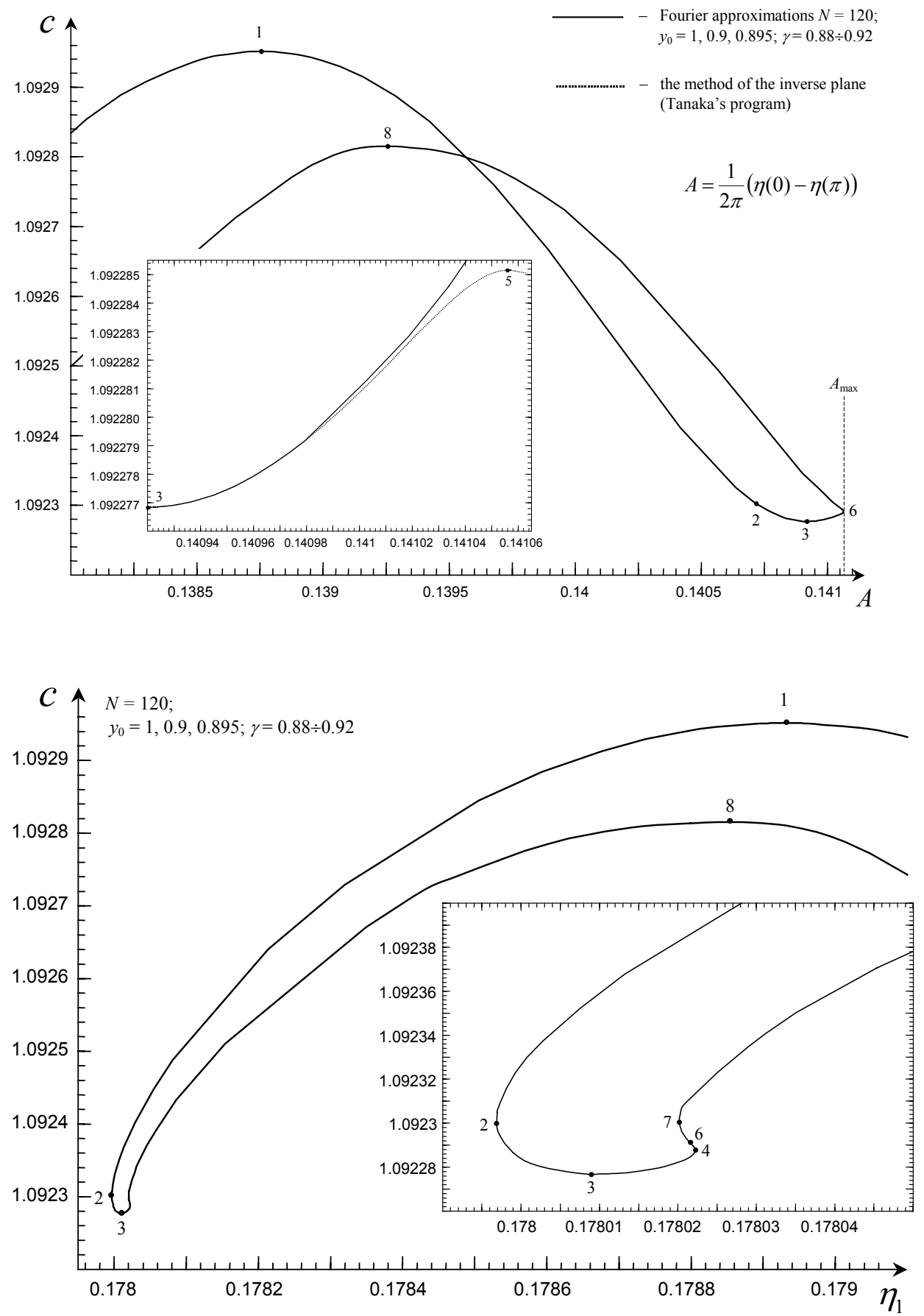

Fig. 2. The dependence of the phase speed $c$ of steep surface waves on their steepness $A$.

Fig. 3. The dependence of the phase speed $c$ of steep surface waves on the first harmonic $\eta_{1}$ of their profile.

Fig. 3 (the curve 4-6-7). As the limiting point 6 is passed in this way, the wave steepness $A$ can again be used as a governing parameter to obtain the whole irregular branch 6-8 shown in Figs. 2 and 3.

While moving along the irregular branch away from the limiting point 6 the accuracy of approximate solutions at fixed $N$ and $M$ drops since the stagnation point settles down deeper into the flow domain. Because of this the branches corresponding to irregular flows in Figs. 2, 3 have not yet stabilized with respect to increasing the truncation numbers $N$ and $M$, although fractional approximations (Eq. 12) in combination with non-linear transformation (Eq. 19) are up to one decimal order more accurate than ordinary Fourier approximations (Eq. 10) when calculating irregular waves. The loop in Fig. 2 still enlarges with increasing $N$, the crosssection point with the Stokes branch moving to the left. On the contrary, the irregular branch 6-7-8 in the dependence $c\left(\eta_{1}\right)$ (see Fig. 3) approaches to the regular branch 1-2-3-4-6 as accuracy is increased. Moreover, the dependences $c(A)$ and $c\left(\eta_{1}\right)$ should actually be much more complicated near 
Table 1. The points of extremums in phase speed $c$, steepness $A(\varepsilon=\pi A)$, and the first harmonic $\eta_{1}$ of the profile for Stokes waves $\left(N=120, M=2.5 N, y_{0}=0.9, \gamma=0.92\right)$

\begin{tabular}{cccccc}
\hline point & extremum & $A$ & $\varepsilon$ & $c$ & $\eta_{1}$ \\
\hline & the first max of $\eta_{1}$ & 0.1351 & 0.424429 & 1.0909437483 & 0.1799822 \\
1 & the first max of $c$ & 0.13875 & 0.435896 & 1.0929513818 & 0.1789318 \\
2 & the first min of $\eta_{1}$ & 0.14072 & 0.442085 & 1.0923021558 & 0.1779969 \\
3 & the first min of $c$ & 0.14092 & 0.442713 & 1.0922768392 & 0.1780099 \\
4 & the second max of $\eta_{1}$ & $\approx 0.141055$ & 0.443137 & $\approx 1.092288$ & 0.1780222 \\
$5^{*}$ & the second max of $c$ & 0.141056 & 0.443141 & 1.0922851495 & \\
6 & max of $A$ (the limiting value) & $\approx 0.141064$ & 0.443166 & $\approx 1.09229$ & 0.1780216 \\
\hline
\end{tabular}

*Results from Tanaka's program

Table 2. The wave speed $c$ and the mean water level $\eta_{0}$ for steep Stokes waves depending on their steepness $A(\varepsilon=\pi A)^{\sharp}$. The maximal relative errors $E r_{\max }$ of corresponding approximate solutions demonstrate great advantage of fractional approximations over ordinary Fourier approximations

\begin{tabular}{|c|c|c|c|c|c|c|c|c|}
\hline \multirow[b]{2}{*}{$A$} & \multirow[b]{2}{*}{$\varepsilon$} & \multicolumn{3}{|c|}{ Ordinary Fourier approximations 1} & \multicolumn{3}{|c|}{ Fractional Fourier approximations ${ }^{2}$} & \multirow{2}{*}{$\begin{array}{c}\text { Tanaka's program } \\
c\end{array}$} \\
\hline & & $c$ & $\eta_{0}$ & $E r_{\max }, \%$ & $c$ & $\eta_{0}$ & $E r_{\max }, \%$ & \\
\hline 0.14 & $\approx 0.439823$ & 1.0926149034 & $-2.8 \cdot 10^{-20}$ & $3.8 \cdot 10^{-10}$ & $1.0926149034^{a}$ & $-2.4 \cdot 10^{-40}$ & $2.9 \cdot 10^{-20}$ & 1.0926149034 \\
\hline 0.1406 & $\approx 0.441708$ & 1.0923377398 & $-5.1 \cdot 10^{-13}$ & $1.6 \cdot 10^{-5}$ & $1.0923377499^{a}$ & $-1.1 \cdot 10^{-22}$ & $3.2 \cdot 10^{-11}$ & 1.0923377499 \\
\hline 0.14092 & $\approx 0.442713$ & 1.09227614 & $-1.9 \cdot 10^{-9}$ & $2.9 \cdot 10^{-3}$ & 1.0922768392 & $-2.0 \cdot 10^{-14}$ & $2.1 \cdot 10^{-6}$ & 1.0922768392 \\
\hline 0.141 & $\approx 0.442965$ & 1.0922815 & $-1.3 \cdot 10^{-8}$ & $9.6 \cdot 10^{-3}$ & 1.0922809 & $-1.7 \cdot 10^{-11}$ & $1.2 \cdot 10^{-4}$ & 1.0922808596 \\
\hline 0.14103 & $\approx 0.443059$ & 1.0922875 & $-2.8 \cdot 10^{-8}$ & $1.9 \cdot 10^{-2}$ & 1.0922841 & $-2.2 \cdot 10^{-10}$ & $5.8 \cdot 10^{-4}$ & 1.0922836847 \\
\hline 0.141056 & $\approx 0.443141$ & 1.0922966 & $-6.1 \cdot 10^{-8}$ & $2.4 \cdot 10^{-2}$ & 1.0922877 & $-2.6 \cdot 10^{-9}$ & $2.5 \cdot 10^{-3}$ & 1.0922851495 \\
\hline 0.14106 & $\approx 0.443153$ & 1.0922987 & $-7.0 \cdot 10^{-8}$ & $2.6 \cdot 10^{-2}$ & $\begin{array}{l}1.0922886 \\
1.0922871^{b}\end{array}$ & $\begin{array}{l}-4.2 \cdot 10^{-9} \\
-2.3 \cdot 10^{-9}\end{array}$ & $\begin{array}{l}3.3 \cdot 10^{-3} \\
2.2 \cdot 10^{-3}\end{array}$ & 1.0922851047 \\
\hline 0.141064 & $\approx 0.443166$ & 1.0923011 & $-8.1 \cdot 10^{-8}$ & $2.8 \cdot 10^{-2}$ & 1.0922902 & $-9.0 \cdot 10^{-9}$ & $5.2 \cdot 10^{-3}$ & $-^{*}$ \\
\hline & & $\begin{array}{l}{ }^{1} N=250, M \\
{ }^{\sharp} \text { The extremum }\end{array}$ & $\begin{array}{l}=4 \mathrm{~N} \\
\text { in wave speed }\end{array}$ & re bold-faced & $\begin{array}{l}{ }^{2} N=120, M \\
{ }^{a} N=120, M \\
{ }^{b} N=150, M \\
{ }^{*} \text { The maximum }\end{array}$ & $\begin{array}{l}2.5 N, y_{0}=0.9 \\
2 N, \quad y_{0}=1, \\
2.5 N, y_{0}=0.9 \\
\text { eepness in Tana }\end{array}$ & $\begin{array}{l}\gamma=0.92 \\
\gamma=0.9 \\
\gamma=0.92 \\
\text { a's program }\end{array}$ & $A \approx 0.1410635$ \\
\hline
\end{tabular}

the turning point than is obtained at present since the regular branch is expected to have an infinite number of extremums in $c$ and $\eta_{1}$ in the case of being evaluated exactly. Because of this it is also not clear now whether there is a bifurcation to the irregular branch from the Stokes branch. Much more accurate calculations and, therefore, further improvement of the method are necessary to clarify these points and to stabilize the position of the irregular branch.

Thus, our main concern is that irregular flow is an approximate solution, whose accuracy is not sufficient enough to make definite conclusions even when using fractional approximations. Nevertheless, some progress was achieved.

Consider the example of the irregular flow calculated using fractional Fourier approximations at $A=0.14092$ and shown in Fig. 5, with the streamlines mapped outside the flow domain being presented as well. The stagnation point $O_{1}$ (where the streamlines again meet at right angles in agreement with Appendix C) is now inside the flow domain in contrast to the regular flows considered in Sect. 4.1. One can see from Fig. 5 that this stagnation point makes streamlines of the irregular flow be discontinuous near the wave crest. Because of this the wave profile $\eta(\theta)$ (that remains to be a continuous function everywhere) does not coincide in the region close to the wave crest with the streamline $\psi=0$ corresponding to a free surface. It is clear that this turns out to be a numerical inaccuracy. This is due to the Fourier series (Eq. 20) for $\eta(\theta)$ being a single-valued smooth function, which represents an integral characteristic of the flow. On the contrary, the stream function $\psi(\theta, y)$ represents a local characteristic of the flow and is not obligatory a single-valued 
Table 3. The parameters of the irregular flow at $A=0.14092$ for different approximations: $c$ is the wave phase speed; $\eta_{1}$ is the first harmonic of the elevation; $\eta_{0}$ is the mean water level (it should be zero for exact solutions); $E r_{\max }$ is the maximal relative error of an approximate solution; $q(0)-c$ is the velocity at the crest in the wave related frame of reference; $\eta(0)$ is the height of the crest above the still water level; $y_{s}$ is the vertical position of the stagnation point; $\eta(0)-y_{s}$ is the distance of the stagnation point from the wave crest. The same parameters of the regular flow at $A=0.14092$ are also included for comparison*

\begin{tabular}{|c|c|c|c|c|c|c|c|c|}
\hline approximation & $c$ & $\eta_{1}$ & $\eta_{0}$ & $E r_{\max }, \%$ & $q(0)-c$ & $\eta(0)$ & $y_{S}$ & $\eta(0)-y_{s}$ \\
\hline \multicolumn{9}{|c|}{ irregular flow } \\
\hline$N=250, M=3 N^{1}$ & 1.092427 & 0.178169 & $-1.7 \cdot 10^{-6}$ & $1.5 \cdot 10^{-1}$ & 0.0531 & 0.595445 & 0.593830 & 0.001615 \\
\hline$N=100, M=140^{2}$ & 1.092350 & 0.178039 & $-3.9 \cdot 10^{-7}$ & $4.2 \cdot 10^{-2}$ & 0.0470 & 0.595636 & 0.594947 & 0.000689 \\
\hline$N=130, M=180^{2}$ & 1.092330 & 0.178024 & $-2.8 \cdot 10^{-7}$ & $3.1 \cdot 10^{-2}$ & 0.0455 & 0.595652 & 0.595117 & 0.000535 \\
\hline$N=160, M=200^{2}$ & 1.092318 & 0.178017 & $-2.2 \cdot 10^{-7}$ & $2.4 \cdot 10^{-2}$ & 0.0448 & 0.595661 & 0.595221 & 0.000440 \\
\hline \multicolumn{9}{|c|}{ regular flow } \\
\hline$N=120, M=2.5 N^{2}$ & 1.092277 & 0.178010 & $-2.0 \cdot 10^{-14}$ & $2.1 \cdot 10^{-6}$ & -0.0419 & 0.595657 & 0.599019 & -0.003362 \\
\hline
\end{tabular}

dependence. Because of this the streamline $\psi=0$ describes a free surface near the wave crest more adequately than the wave profile $\eta(\theta)$.

What is the nature of this inaccuracy? It is seen from Fig. 5 that the profile of the irregular flow oscillates while approaching the wave crest, where a prominent peak (an overshoot) forms. This highly resembles the Gibbs phenomenon when (i) a discontinuous function or (ii) a continuous function with discontinuous derivatives (a weak discontinuity) are approximated by a truncated set of continuous functions (see, e.g. Arfken and Weber, 1995). In both cases, the Gibbs phenomenon is an excellent indicator of a singularity. Thus, irregular waves correspond to singular solutions of the equations of motion.

The example corresponding to the case (i) is given in Appendix E, where truncated Fourier series of a function with infinite discontinuity are demonstrated to exhibit typical Gibbs oscillations and an overshoot that moves to infinity as accuracy is improved (see Fig. E1). In contrast to this example, however, the overshoot in the profile of irregular waves has an almost fixed vertical position and shrinks both in vertical and horizontal scales as numerical accuracy is improved when proceeding from the ordinary to fractional Fourier approximations, as one can see from Fig. 6. The same situation is observed for regular Stokes waves very close to the Stokes corner flow having a sharp $120^{\circ}$ corner at the crest (a discontinuous first derivative) that corresponds to the case (ii) of weak discontinuity. Such waves also exhibit Gibbs oscillations when being approximated numerically, as was revealed by Chandler and Graham (1993) from the analysis of Nekrasov's integral equation. The similar example obtained using fractional and ordinary Fourier expansions is presented in Fig. 7 for the Stokes wave at $A=0.14106(\approx 99.9975 \%$ of the limiting steepness $A \approx 0.1410635)$. The Gibbs phe- nomenon is distinctly observed and the overshoot shrinks both in vertical and horizontal scales with increasing the accuracy of approximations in the same way as the overshoot of the irregular wave presented in Fig. 6 . In the case of the limiting Stokes wave, the overshoot is absent in the exact solution that has a sharp $120^{\circ}$ corner at the crest, that is, it shrinks completely. Thus, the conclusion can be made that the overshoot in the profile of irregular waves should to all appearance also shrink into a single point when increasing accuracy further. What would the wave profile look like then?

To answer this question let us analyse how the parameters of an approximate irregular flow at fixed steepness $A=0.14092$ depend on improving numerical accuracy using Table 3 . The distance $\eta(0)-y_{s}$ between the wave crest and the stagnation point becomes approximately four times as small when using more accurate fractional approximations instead of ordinary Fourier approximations, the horizontal and vertical dimensions of the overshoot both becoming approximately five times as small. This correlates with decreasing the maximal relative error $E r_{\max }$ of the solutions approximately by a factor of six. The particle velocity at the crest $q(0)-c$ in the wave related frame of reference also decreases but less rapidly than the relative error $E r_{\max }$ of the corresponding solutions. On the contrary, the wave height $\eta(0)$ quickly stabilizes with increasing accuracy and seems not to tend to the height of the Stokes corner flow.

Although the data presented do not give the final understanding of irregular flows at $N \rightarrow \infty$, the following assumption seems to be quite reasonable. The overshoot shrinks into a single point and the particle velocity at the crest $q(0)-c$ drops to zero. The stagnation point settles down at the wave crest and the horn-like separatrices merge forming a flow with a sharp corner at the crest that holds for any steepness $A$ up to the limiting value, the wave pro- 

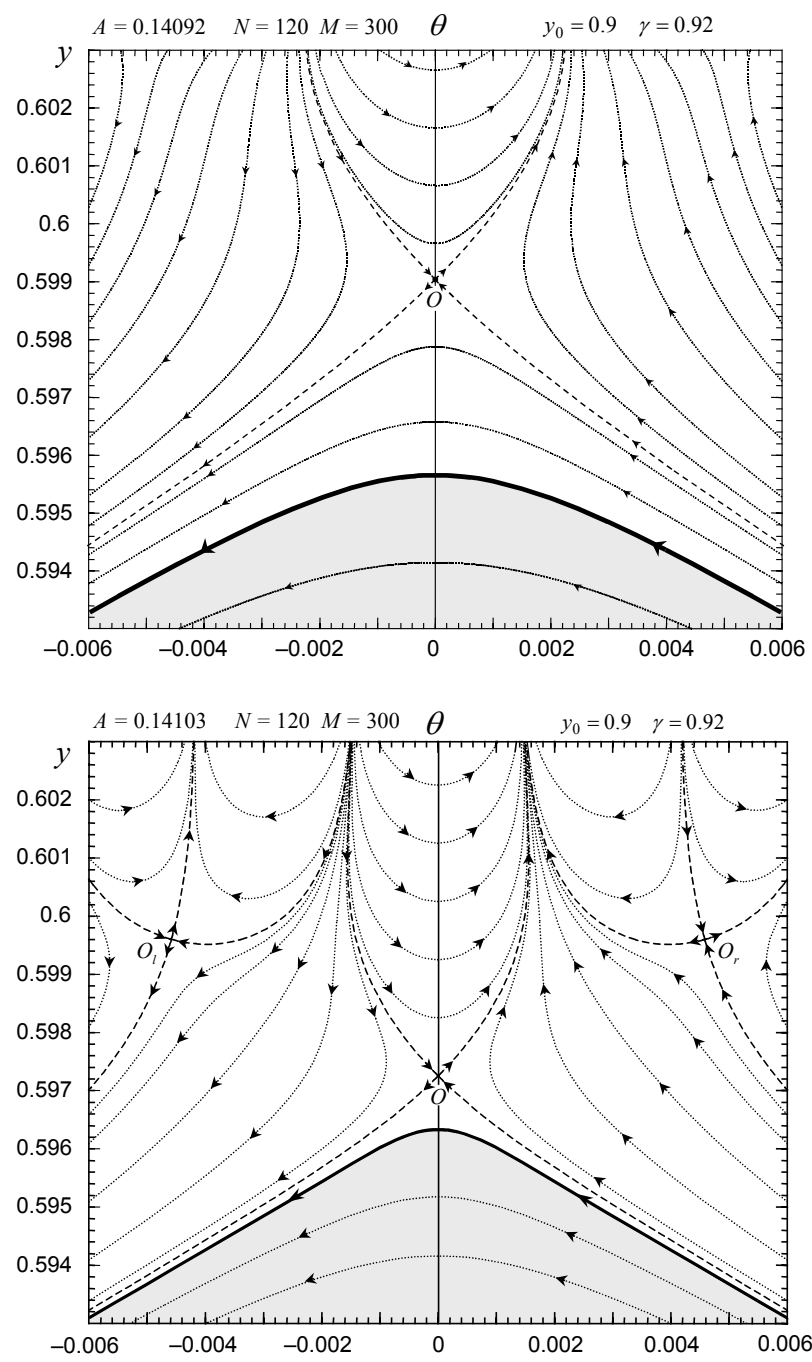

Fig. 4. The regular flow in the crest region of almost highest Stokes waves at two different values of the wave steepness, in the wave related frame of reference, the streamlines mapped outside the domain filled by fluid being presented as well.

file coinciding with the streamline $\psi=0$ all over the free surface. The Stokes theorem about a $120^{\circ}$ corner flow (see Appendix D) is generally valid for corner flow independently of the wave amplitude. Therefore, irregular waves to all appearance turn out to approximate a family of sharp-crested waves with $120^{\circ}$ corner at the crest like the limiting Stokes wave but of lesser steepness. Sharp-crested corner flows and regular Stokes flows both tend to the Stokes corner flow as the wave steepness is increased. Moreover, the additional stagnation points $O_{l}$ and $O_{r}$ in Fig. 5 approach to the central stagnation point $O_{1}$ as numerical accuracy is improved. Therefore, the stagnation point at the crest of sharp-crested waves should to all appearance also be formed from several coalescing singularities similar to the limiting Stokes wave.

Although irregular flows with stagnation point inside the flow domain are only approximate numerical solutions, they can be used for simulating the process of wave breaking. One can see from Fig. 5 that the particles from the near-surface layer of the irregular flow are accelerated to velocities greater than the wave phase speed when approaching the crest. As a result, they form the upward jet emanating from the front face of the wave. The acceleration of particles at the base of the jet ranges from $2.5 g$ at $\theta=0.001$ to $6 g$ at the wave crest. Such large accelerations of the water rising up the front of the wave into the jet are in fact known to occur in breaking waves (see Banner and Peregrine, 1993), where the typical maxima in accelerations obtained from detailed unsteady numerical computations were reported to be around $5 g$. The following subsequent unsteady evolution of irregular flow is expected. The particles with velocities greater than the wave speed will all be jetted out away from the fluid and the crest will break if their is no external influx into the flow domain from the left downward jet. After finishing this non-stationary process the flow will become regular and of lesser steepness. This resembles the recurrence phenomenon observed by LonguetHiggins and Dommermuth (1997) when computing unsteady non-linear development of the crest instabilities of almost highest Stokes waves resulting in a smooth transition to a periodic wave of lower amplitude. The appearance of irregular flows in their numerical scheme may be a reason for this phenomenon.

\section{Conclusions}

Fractional Fourier approximations for the velocity potential in combination with non-linear transformation of the horizontal scale, which concentrates a numerical emphasis on the crest region, turned out to be much more efficient than ordinary Fourier approximations when computing both steep regular and irregular flows. Nevertheless, further improvement of the numerical algorithm is necessary to achieve the accuracy of Tanaka's method when calculating almost limiting Stokes waves and to attain the final understanding of irregular flows. One of the possible ways is to use the following multi-term fractional expansion with several different parameters $y_{k}$ :

$$
R\left(\theta, y ;\left\{y_{k}\right\}\right)=\sum_{k=0}^{K} \sum_{n=0}^{N_{k}} \frac{\alpha_{n}^{(k)}}{\left(\exp \left(-y_{k}\right)-\exp (-y-i \theta)\right)^{n}} .
$$

Although the proposed approach was formulated in the framework of the canonical model for infinite depth, its practical application is much broader. Gandzha et al. (2003) successfully employed fractional approximations for computing gravity-capillary waves. When $y_{0}$ is located inside the flow domain fractional approximations may be applied for calculating vortex structures and solitary waves. The latter possibility was realized by Clamond (2003) using his renormalized cnoidal wave approximation (the first term of the fractional Fourier approximation). He computed an algebraic solitary wave on deep water and traced how it changes after taking into account surface tension (it is not known, however, how this algebraic solution depends on picking up higher 


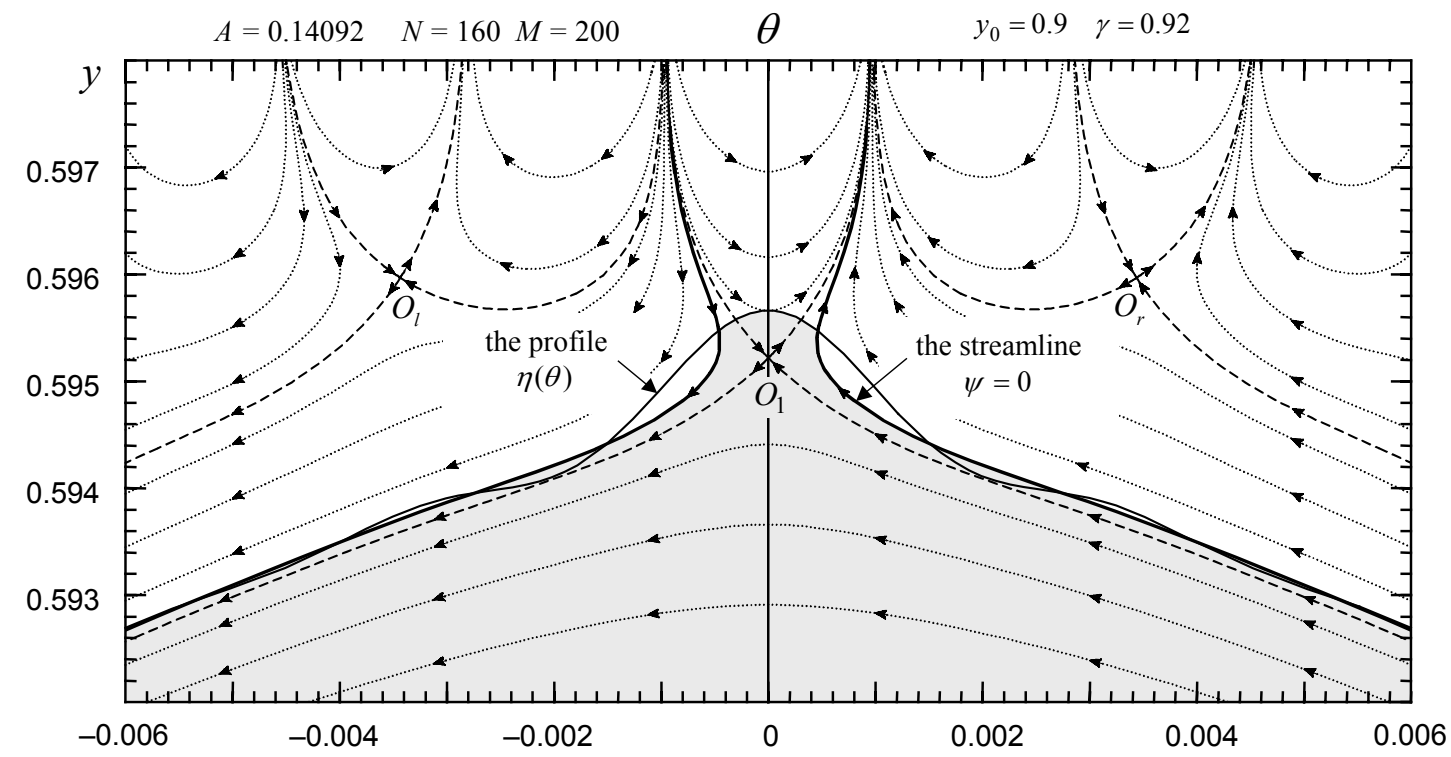

Fig. 5. The profile and streamlines of the irregular flow near the wave crest, in the wave related frame of reference, the streamlines mapped outside the domain filled by fluid being presented as well.

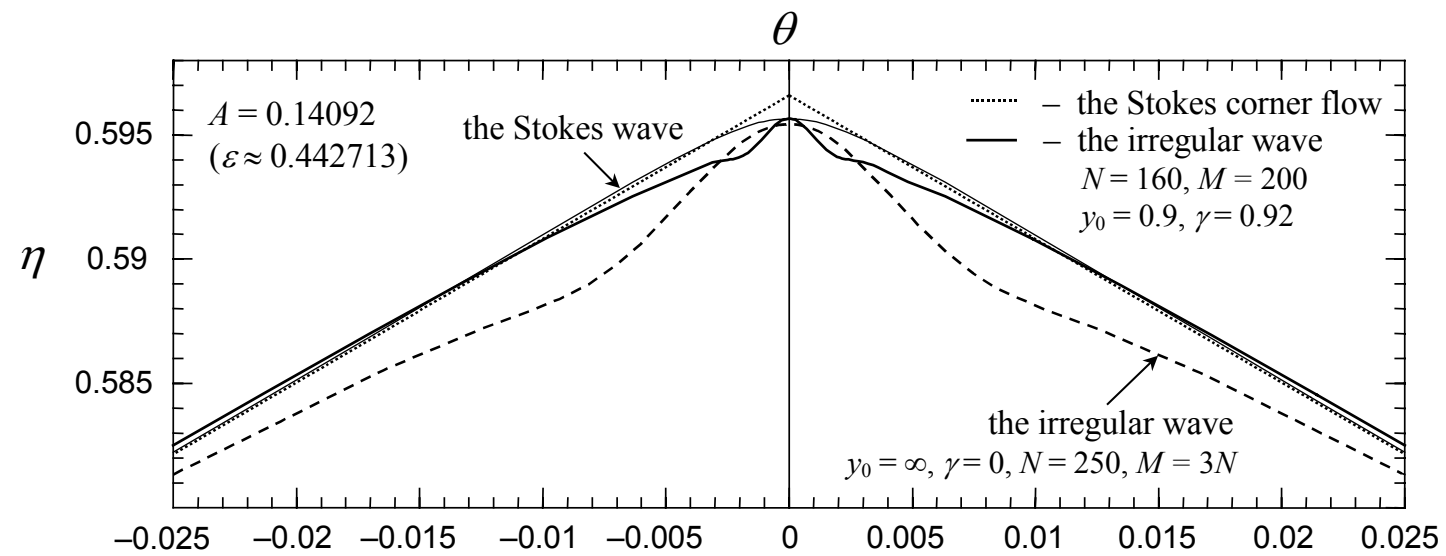

Fig. 6. The behavior of the overshoot (the Gibbs phenomenon) in the profile of the irregular wave when improving numerical accuracy due to proceeding from the ordinary to fractional Fourier approximations. The profile of the Stokes wave of the same steepness is also included for comparison.

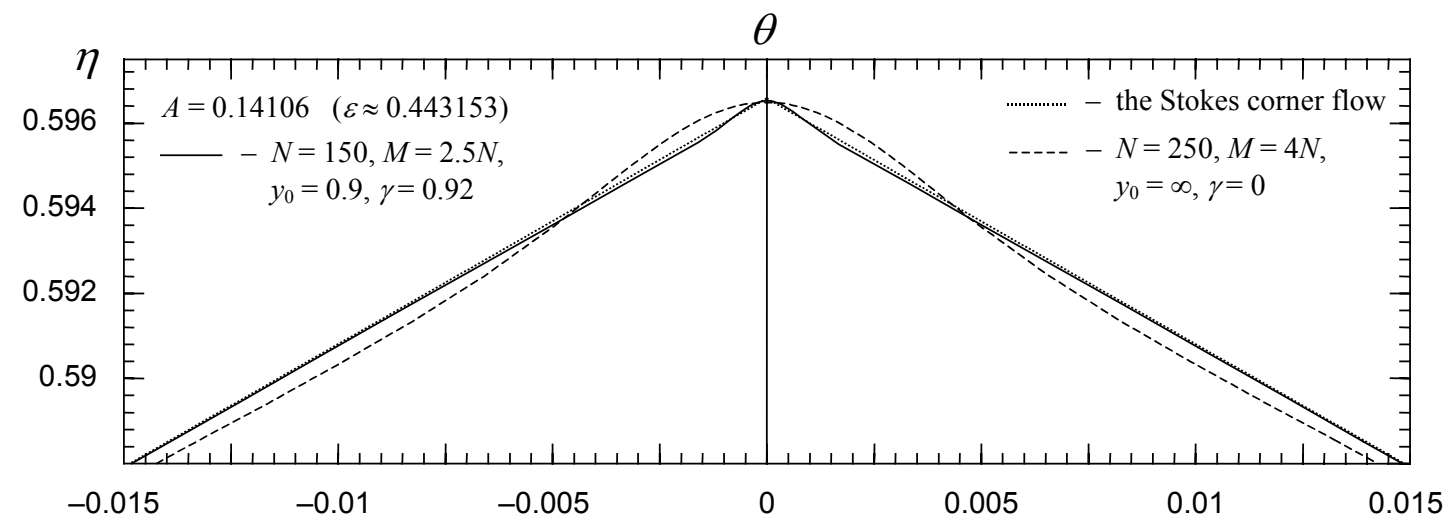

Fig. 7. The Gibbs phenomenon in the approximations to the regular Stokes wave very close to the Stokes corner flow. 
terms of the fractional expansion). In the case of finite depth $h$, the fractional Fourier expansion will be as follows:

$$
\begin{gathered}
R\left(\theta, y ; y_{0}\right)=\sum_{n=0}^{N}\left(\frac{\alpha_{n}^{(+)}}{\left(\exp \left(-y_{0}\right)-\exp (-y-h-i \theta)\right)^{n}}-\right. \\
\frac{\alpha_{n}^{(-)}}{\left.\left(\exp \left(-y_{0}\right)-\exp (y+h+i \theta)\right)^{n}\right)} .
\end{gathered}
$$

Finally, fractional expansions may also be generalized to the case of 3-D waves and non-ideal fluid.

Fractional approximations allowed us to gain more detailed knowledge about the properties of irregular flows. Irregular waves were proved to correspond to singular solutions of the equations of motion. Because of this their existence does not contradict to the uniqueness theorem of Garabedian (1965) since it deals with regular continuous solutions only. The profiles of exact solutions (sharp-crested waves) corresponding to irregular waves seem to have a sharp $120^{\circ}$ corner at the crest (a discontinuous first derivative) like the limiting Stokes wave but of lesser amplitude. Such solutions are also known to occur in the physics of shock waves, where they are called the surfaces of weak discontinuity (see Landau and Lifshitz, 1995). Further analysis, however, should be carried out to make a final conclusion. One of the possible ways is to investigate how an approximate irregular flow depends on taking into account surface tension and to make a comparison with new limiting forms for gravity-capillary waves recently obtained by Debiane and Kharif (1996).

To conclude note that the formation of jets from irregular progressive waves resembles the occurence of vertical jets with sharp-pointed tips from standing gravity waves forced beyond the maximum height, as has recently been reported by Longuet-Higgins (2001).

Acknowledgements. We are grateful to Prof. M. Tanaka for being so kind to place at our disposal his program for calculating Stokes waves. We express thanks and appreciation to Professors D.H. Peregrine, C. Kharif, V.I. Shrira, and Dr. D. Clamond for many valuable advices and fruitful discussions. The research of I. Gandzha has been supported by INTAS Fellowship YSF 2001/2-114.

\section{Appendix A The relations between the Fourier coeffi- cients in the $\theta$ - and $\chi$-spaces}

Taking into account nonlinear transformation (19) the coefficients in Fourier series (18) and (20) are connected as follows

$$
\begin{aligned}
& \eta_{0}=\eta_{0}^{(\gamma)}-\gamma \eta_{1}^{(\gamma)} \\
& \eta_{n}=\frac{1}{n} \sum_{n_{1}=1}^{M} n_{1} \eta_{n_{1}}^{(\gamma)}\left(J_{n-n_{1}}(n \gamma)-J_{n+n_{1}}(n \gamma)\right)
\end{aligned}
$$

$n=\overline{1, \infty} ; J_{n}(z)$ being the Bessel function of the first kind.

\section{Appendix B The Jacoby matrix}

The Jacoby matrix is composed of the coefficients at the infinitesimal variations $\delta c, \delta \eta_{0}^{(\gamma)}, \delta \eta_{n_{1}}^{(\gamma)}\left(n_{1}=\overline{2, M}\right), \delta \alpha_{n_{1}}$ $\left(n_{1}=\overline{1, N}\right)$ of the unknown variables in the following variations of equations (21) and (22):

$$
\begin{aligned}
& \delta \mathcal{D}_{n}=\sum_{n_{1}=1}^{N} \alpha_{n, n_{1}}^{22} \delta \alpha_{n_{1}}+\sum_{n_{1}=0}^{M} \alpha_{n, n_{1}}^{21} \delta \eta_{n_{1}}^{(\gamma)}+2 c d_{n} \delta c ; \\
& \delta \mathcal{K}_{n}=\sum_{n_{1}=1}^{N} \alpha_{n, n_{1}}^{12} \delta \alpha_{n_{1}}+\sum_{n_{1}=0}^{M} \alpha_{n, n_{1}}^{11} \delta \eta_{n_{1}}^{(\gamma)}
\end{aligned}
$$

where

$$
\begin{aligned}
\alpha_{n, n_{1}}^{11} & =\alpha_{n-n_{1}}^{11}+\alpha_{n+n_{1}}^{11}, \alpha_{n, 0}^{11}=\alpha_{n}^{11} ; \\
\alpha_{n, n_{1}}^{12} & =2 t_{n}^{\left(n_{1}\right)} ; \quad \alpha_{n}^{11}=2 \sum_{n_{1}=1}^{N+1} \beta_{n_{1}} t_{n}^{\left(n_{1}\right)}-\delta_{n, 0} ; \\
\alpha_{n, n_{1}}^{21}= & \alpha_{n-n_{1}}^{21}+\alpha_{n+n_{1}}^{21}, \alpha_{n, 0}^{21}=\alpha_{n}^{21} ; \\
\alpha_{n}^{21}= & 2 c^{2} \sum_{n_{1}=1}^{N+1} \beta_{n_{1}}\left(n_{1}\left(t_{n}^{\left(n_{1}\right)}-t_{n}^{\left(n_{1}+1\right)} e^{-y_{0}}\right)-\right. \\
\sum_{n_{2}}=n_{1} & \left(2-\delta_{n_{1}, n_{2}}\right) \beta_{n_{2}}\left(\left(n_{1}+n_{2}\right) t_{n}^{\left(n_{1}, n_{2}\right)}-\right. \\
n_{1} & \left.t_{n}^{\left(n_{1}+1, n_{2}\right)} e^{-y_{0}}-n_{2} t_{n}^{\left(n_{1}, n_{2}+1\right)} e^{\left.-y_{0}\right)}\right)-\delta_{n, 0} ; \\
\alpha_{n, n_{1}}^{22}= & 2 n_{1} c^{2}\left(t_{n}^{\left(n_{1}\right)}-t_{n}^{\left(n_{1}+1\right)} e^{-y_{0}}-\right. \\
& \left.2 \sum_{n_{2}=1}^{N+1} \beta_{n_{2}}\left(t_{n}^{\left(n_{1}, n_{2}\right)}-t_{n}^{\left(n_{1}+1, n_{2}\right)} e^{\left.-y_{0}\right)}\right)\right) .
\end{aligned}
$$

Note that the variation $\delta \eta_{1}^{(\gamma)}$ should be expressed in terms of the rest variations $\delta \eta_{n}^{(\gamma)}, n=\overline{2, M}$ using expression (25) if the governing parameter is the steepness $A$ or the relation (A1) at $n=1$ if the governing parameter is the first harmonic $\eta_{1}$ of the elevation in the $\theta$-space.

\section{Appendix C The stagnation point}

The point in the flow field, where fluid particles are motionless in the wave related frame of reference, is called the stagnation point. For symmetric regular/irregular flows, the stagnation point is located above/below the wave crest outside/inside the flow domain on the axis $\theta=0$. Then its vertical position $y_{s}$ is determined as follows

$\Phi_{\theta}\left(0, y_{s}\right)=c$.

To find the velocity field $\Phi_{\theta}(\theta, y), \Phi_{y}(\theta, y)$ in the infinitesimal vicinity $\theta=\tilde{\theta}(\tilde{\theta} \rightarrow 0), y=y_{s}+\tilde{y}(\tilde{y} \rightarrow 0)$ of the stagnation point it is sufficient to linearize there the expansions (28), (29) that represent exact velocity field at $N \rightarrow \infty$. For this, one should linearize the functions 


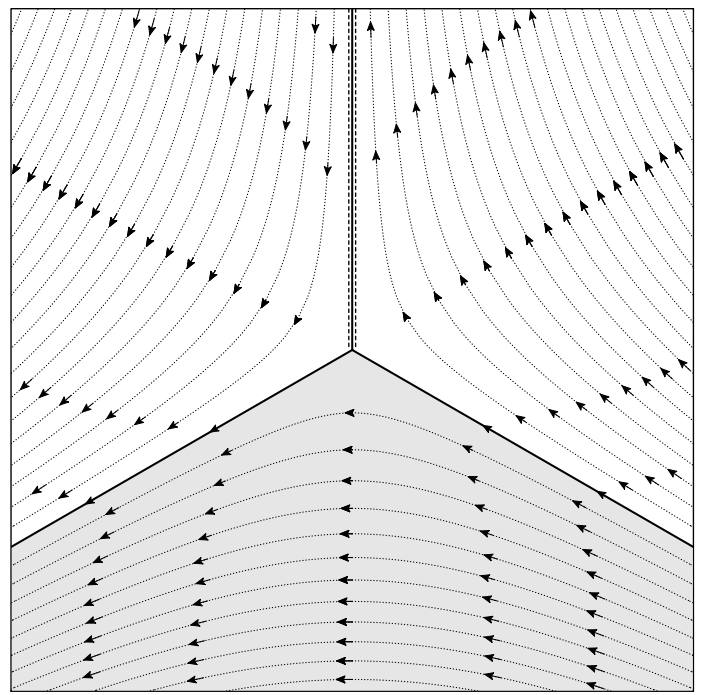

Fig. D1. A local $120^{\circ}$ corner flow, the cut of the complex plane being dashed.

$T^{n}(\theta, y)$ around the stagnation point as follows

$$
\begin{aligned}
T(\theta, y) & =T\left(y_{s}+\tilde{y}+i \tilde{\theta}\right)=T\left(y_{s}\right)+T^{\prime}\left(y_{s}\right)(\tilde{y}+i \tilde{\theta}) \\
T^{n}(\theta, y) & =T^{n}\left(y_{s}\right)+n T^{\prime}\left(y_{s}\right) T^{n-1}\left(y_{s}\right)(\tilde{y}+i \tilde{\theta}) .
\end{aligned}
$$

Then, after taking into account condition (C1), the Eqs. (5) for particle trajectories attain the following form in the vicinity of the stagnation point:

$$
\frac{d \tilde{\theta}}{d t}=a \tilde{y}, \quad \frac{d \tilde{y}}{d t}=a \tilde{\theta} ; a=2 c \sum_{n=1}^{\infty} n \beta_{n} T^{\prime}\left(y_{s}\right) T^{n-1}\left(y_{s}\right) .
$$

Therefore, the equations for the streamlines are $\tilde{\theta}= \pm \tilde{y}$. Actually, this is a direct consequence of the fact that any solution to the Laplace equation (1) should depend not on the variables $(\theta, y)$ separately but on their combination $y+i \theta$.

Thus, the streamlines meet at right angles $\left(90^{\circ}\right)$ at the stagnation point. This fact is valid for any flow provided that the stagnation point and the wave crest do not merge. Otherwise, $y_{s}=\eta(0) ; \tilde{\theta}$ and $\tilde{x}$ are not independent variables, and linearization $(\mathrm{C} 2)$ is not valid. In this case, the streamlines turn out to meet at $120^{\circ}$ angle (see Appendix D), as was at first shown by Stokes (1880).

\section{Appendix D The Stokes theorem}

Stokes rigourously showed that the only possible local crest singularity of a steady wave is a corner of $120^{\circ}$. Hereafter we emphasize that Stokes theorem is generally valid for a wave of any amplitude, not only for the limiting wave.

Choose the origin of the wave related frame with upward vertical axis $y$ and left-to-right horizontal axis $\theta$ at the wave crest. Let $\phi(\theta, y)$ be the velocity potential in the wave related frame. Choose the stream function $\psi(\theta, y)$ to be
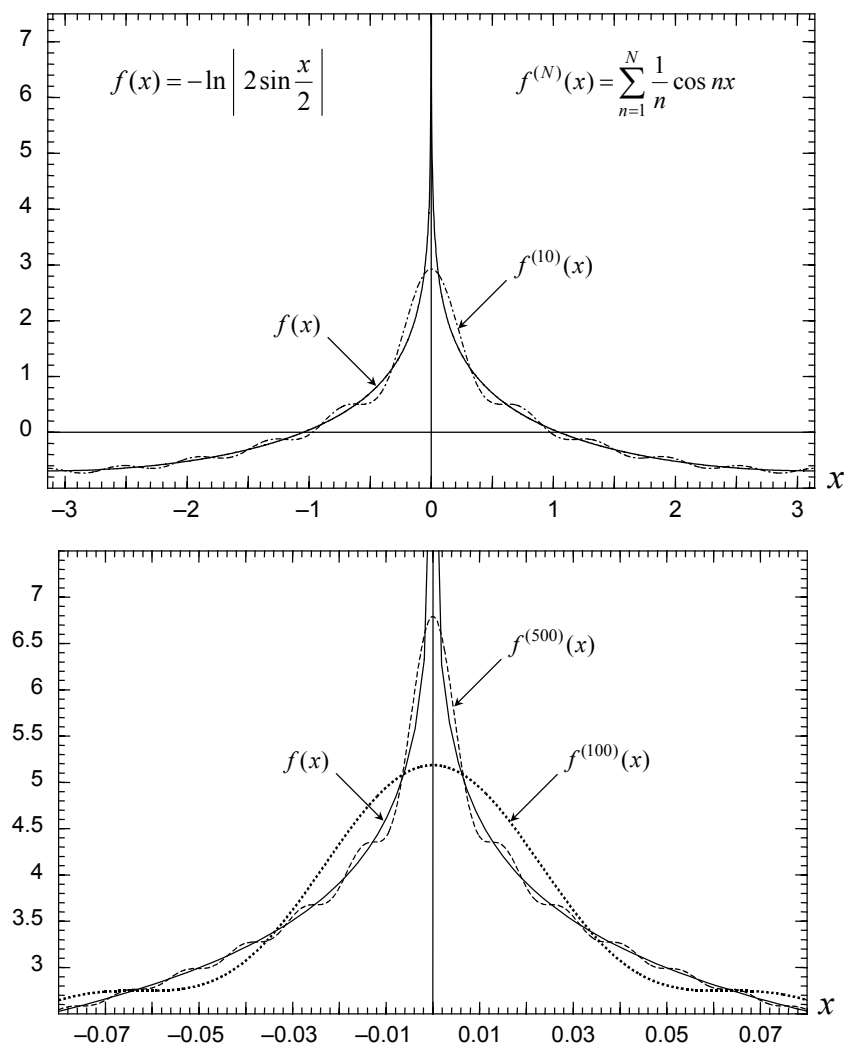

Fig. E1. The truncated Fourier series of the discontinuous function. The Gibbs phenomenon.

zero at the free surface. In terms of the complex coordinate $z=\theta+i y=r \exp (i \varphi)$ and the complex potential $w(z)=\phi+i \psi$, the Bernoulli equation (2) is as follows:

$\left|\frac{\mathrm{d} w}{\mathrm{~d} z}\right|^{2}+2 \operatorname{Im} z=0$.

The complex potential for a flow including a sharp corner with stagnation point is described by the following function:

$w(z)=A z^{n}=|A| r^{n} \exp \left(i n \varphi+i \varphi_{A}\right)$,

where $-3 \pi / 2<\varphi<\pi / 2, \varphi=\pi / 2$ being the cut of the complex plane. Substitution of (D2) into the Bernoulli equation (D1) yields $n=3 / 2$. Note that (D2) is only locally valid being only the first term in an expansion about the corner. Further terms include the powers of irrational order as was established by Grant (1973) and Norman (1974).

The condition that $\psi=0$ at the corner slopes yields (the corner angle is $2 \alpha$ ):

$\sin \left(\frac{3}{2}\left(\alpha-\frac{\pi}{2}\right)+\varphi_{A}\right)=0 \Rightarrow \frac{3}{2}\left(\alpha-\frac{\pi}{2}\right)+\varphi_{A}=\pi$,

$\sin \left(\frac{3}{2}\left(-\alpha-\frac{\pi}{2}\right)+\varphi_{A}\right)=0 \Rightarrow \frac{3}{2}\left(-\alpha-\frac{\pi}{2}\right)+\varphi_{A}=0$.

Therefore $\alpha=\pi / 3, \varphi_{A}=5 \pi / 4$, and the corner angle is $120^{\circ}$. The corresponding corner flow is shown in Fig. D1.

Thus, if the wave crest has a corner it must be of $120^{\circ}$ independently of the wave amplitude. Nevertheless, the only 
wave known to exhibit such a corner flow is the Stokes wave of limiting amplitude. It seems that irregular waves reveal a family of similar corner flows with amplitudes less than that of the limiting Stokes wave. The Stokes corner flow seems to be formed due to merging the stagnation points of regular and irregular flows at limiting amplitude.

\section{Appendix E The Gibbs phenomenon}

Consider the following $2 \pi$-periodic function

$f(x)=-\ln \left|2 \sin \frac{x}{2}\right|$

with infinite discontinuity at $x=2 \pi k, k \in \mathbb{Z}$. This function constitutes a part of the kernel of Nekrasov's integral equation (see Chandler and Graham, 1993). The truncated Fourier series of the function $f(x)$ have the following form (see Arfken and Weber, 1995):

$f^{(N)}(x)=\sum_{n=1}^{N} \frac{1}{n} \cos (n x), \quad f(x)=\lim _{N \rightarrow \infty} f^{(N)}(x)$.

In this case, the discontinuous function $f(x)$ is approximated by the continuous functions $f^{(N)}(x)$. One can see from Fig. E1 that instead of infinite discontinuities, the functions $f^{(N)}(x)$ have rounded peaks (overshoots) with symmetric oscillatory tails that descend as the distance from the point of discontinuity increases. This is the well known Gibbs phenomenon, which always takes place when approximating discontinuous functions by the truncated Fourier series (see Arfken and Weber, 1995). As the number $N$ is increased, the functions $f^{(N)}(x)$ approximate the function $f(x)$ more precisely. The peak moves upwards and the oscillatory tails move closer to the point of discontinuity, their amplitude and period decreasing. Nevertheless, the height of the peak (the vertical distance between the point $x=0$ and the point, where the oscillatory tail initiates) remains almost constant with increasing $N$. Because of this the truncated Fourier series representation remains unreliable in the vicinity of a discontinuity even for high enough $N$.

\section{References}

Arfken, G. B. and Weber, H. J.: Mathematical Methods for Physicists, Academic Press, London, 1995.

Banner, M. L. and Peregrine, D. H.: Wave breaking in deep water, Ann. Rev. Fluid Mech., 25, 373-397, 1993.

Chandler, G. A. and Graham, I. G.: The computation of water waves modelled by Nekrasov's equation, SIAM J. Numer. Anal., 30, 1041-1065, 1993.

Chen, B. and Saffman, P. G.: Numerical evidence for the existence of new types of gravity waves of permanent form on deep water, Stud. Appl. Math., 62, 1-21, 1980.

Clamond, D.: Steady finite-amplitude waves on a horizontal seabed of arbitrary depth, J. Fluid Mech., 398, 45-60, 1999.

Clamond, D.: Cnoidal type surface wave in deep water, J. Fluid Mech., 489, 101-120, 2003.
Cokelet, E. D.: Steep gravity waves in water of arbitrary uniform depth, Philos. Trans. Roy. Soc. London, 286, 183-221, 1977.

Debiane, M. and Kharif, C.: A new limiting form for steady periodic gravity waves with surface tension on deep water, Phys. Fluids, 8, 2780-2782, 1996.

Gandzha, I. S., Lukomsky, V. P., Lukomsky, D. V., Debiane, M., and Kharif, C.: Numerical evidence for the existence of a new type of steady gravity waves on deep water, in: Geophys. Res. Abstracts, vol. 4, pp. A-01 347, EGS 27th General Assembly, Nice, France, 2002.

Gandzha, I. S., Lukomsky, V. P., Tsekhmister, Y. V., and Chalyi, A. V.: Comparison of ordinary and singular Fourier approximations for steep gravity and gravity-capillary waves on deep water, in Geophys. Res. Abstracts, vol. 5, pp. A-12 192, EGS-AGUEUG Joint Assembly, Nice, France, 2003.

Garabedian, P. R.: Surface waves of finite depth, J. Analyse Math., $14,161,1965$

Grant, M. A.: The singularity at the crest of a finite amplitude progressive Stokes wave, J. Fluid Mech., 59, 257-262, 1973.

Hamming, R. W.: Numercal Methods, Mc Graw-Hill, New York San Francisco - Toronto - London, 1962.

Jillians, W. J.: The superharmonic instability of Stokes waves in deep water, J. Fluid Mech., 204, 563-579, 1989.

Landau, L. D. and Lifshitz, E. M.: Fluid Mechanics (Course of Theoretical Physics, Vol. 6), Pergamon Press, New York, 1995.

Longuet-Higgins, M. S.: Integral properties of periodic gravity waves of finite amplitude, Proc. Roy. Soc. London, A342, 157174, 1975.

Longuet-Higgins, M. S.: Bifurcation and instability in gravity waves, Proc. Roy. Soc. London, A403, 167-187, 1986.

Longuet-Higgins, M. S.: Asymptotic forms for jets from standing waves, J. Fluid Mech., 447, 287-297, 2001.

Longuet-Higgins, M. S. and Cleaver, R. P.: Crest instabilities of gravity waves. Part 1 . The almost highest wave, J. Fluid Mech., 258, 115-129, 1994.

Longuet-Higgins, M. S. and Dommermuth, D. G.: Crest instabilities of gravity waves. Part 3. Nonlinear development and breaking, J. Fluid Mech., 336, 33-50, 1997.

Longuet-Higgins, M. S. and Fox, M. J. H.: Theory of the almost highest wave: the inner solution, J. Fluid Mech., 80, 721-741, 1977.

Longuet-Higgins, M. S. and Fox, M. J. H.: Theory of the almost highest wave. Part 2. Matching and analytic extension, J. Fluid Mech., 85, 769-786, 1978.

Longuet-Higgins, M. S. and Tanaka, M.: On the crest instabilities of steep surface waves, J. Fluid Mech., 336, 51-68, 1997.

Longuet-Higgins, M. S., Cleaver, R. P., and Fox, M. J. H.: Crest instabilities of gravity waves. Part 2. Matching and asymptotic analysis, J. Fluid Mech., 259, 333-344, 1994.

Lukomsky, V. P., Gandzha, I. S., and Lukomsky, D. V.: Computational analysis of the almost-highest waves on deep water, Comp. Phys. Comm., 147, 548-551, 2002a.

Lukomsky, V. P., Gandzha, I. S., and Lukomsky, D. V.: Steep sharp-crested gravity waves on deep water, Phys. Rev. Lett., 89, $164502,2002 b$.

Lukomsky, V. P., Gandzha, I. S., Lukomsky, D. V., Tsekhmister, Y. V., and Chalyi, A. V.: Computational analysis of periodic gravity waves on a free water surface in the vicinity of limiting steepness, in Bull. Amer. Phys. Soc., vol. 45(5), pp. 34-35, Conference on Computational Physics, San Diego, California, 2002c.

Norman, A. C.: Expansions for the shape of maximum amplitude 
Stokes waves, J. Fluid Mech., 66, 261-265, 1974.

Stokes, G. G.: On the theory of oscillatory waves, Camb. Phil. Soc. Trans., 8, 441-455, 1847.

Stokes, G. G.: Considerations relative to the greatest height of oscillatory waves which can be propagated whithout change of form, Math. Phys. Papers, 1, 225-228, 1880.
Tanaka, M.: The stability of steep gravity waves, J. Phys. Soc. Japan, 52, 3047-3055, 1983.

Tanaka, M.: The stability of steep gravity waves. Part 2, J. Fluid Mech., 156, 281-289, 1985.

Tanaka, M.: The stability of solitary waves, Phys. Fluids, 29, 650655, 1986. 